\title{
Mathematical Analysis of Harvested Predator-Prey System with Prey Refuge and Intraspecific Competition
}

\author{
Alanus Mapunda ${ }^{1^{*}}$ and Thadei Sagamiko ${ }^{2}$ \\ ${ }^{l}$ Department of Mathematics and Statistics, Mbeya University of Science and Technology, \\ P. O. Box 131, Mbeya Tanzania \\ ${ }^{2}$ Department of Physics, Mathematics and Informatics, Dar es Salaam University College \\ of Education, University of Dar es Salaam, P. O. Box 2329, Dar es Salaam Tanzania \\ "Corresponding author e-mail: alanusmapunda9@gmail.com \\ E-mail co-author: tsagamiko@gmail.com
}

Received 13 Jan 2021, Revised 12 May 2021, Accepted 19 May 2021, Published May 2021

DOI: https://dx.doi.org/10.4314/tjs.v47i2.28

\begin{abstract}
In this paper, a predator-prey relationship in the presence of prey refuge was studied. The analysis of the dependence of locally stable equilibrium points on the parameters of the problem was carried out. Bifurcation and limit cycles for the model were analyzed to show the dynamical behaviour of the system. The results showed that the system is stable at a constant prey refuge $m=$ 0.3 and prey harvesting rate $H=0.3$. However, increasing $m$ and decreasing $H$ or vice versa, the predator-prey system remains stable. It was further observed that for a constant prey refuge $m \geq$ 0.78 , the predator population undergoes extinction. Therefore, $m$ was found to be a bifurcation parameter and $m=0.78$ is a bifurcation value.
\end{abstract}

Keywords: Prey refuge, bifurcation, harvesting, intraspecific competition, phase portrait.

\section{Introduction}

The dynamic relationship between predators and their preys has been, and continue to be, one of the most important topics of study in ecology and mathematical ecology due to its universal existence and importance (Berryman 1992, Mapunda et al 2018). As species interact, they compete amongst themselves for resources and space. They are doing so either under intraspecific competition or interspecific competition (Schwinning and Kelly 2013). A Holling type I functional response assumes that there is no satiation (linear relationship) rather predators keep on feeding until the prey population goes to extinction or remains with few individuals to make preying difficult (Dawes and Souza 2013). This situation leads to competition for resources among the predators. A prey refuge is one of the strategies that can be used to protect preys from over-predation (Sagamiko et al. 2015). Increasing prey refuge implies increasing prey density (Kar 2005).

Bifurcation is a sudden change of stability behaviour of a dynamical system at equilibrium as an equilibrium parameter changes. For complex dynamical systems, bifurcation theory and stability analysis are used for investigating their dynamical behaviour without determining explicitly the solutions of their governing equations for various initial and boundary conditions (Stefanou and Alevizos 2016).

Much work has been done on predator-prey systems incorporating prey refuge and species competition as pointed out by Majeed (2018), Kar (2005), Kar (2006), Das et al. (2013) and 
Sagamiko et al. (2015). Majeed (2018), studied the dynamics of a prey-predator model with refuge and stage structures in both populations, Sagamiko et al. (2015) considered a threatened prey-predator system with prey refuge in the Serengeti ecosystem, Kar (2005) worked on the stability analysis of a prey-predator model incorporating a prey refuge, Kar and Chakraborty (2010) explored the effort dynamics in a prey-predator model with harvesting in which competition among predators was considered. In particular, Kar (2006) and Das et al. (2013) investigated the dynamics of exploited prey-predator with constant prey refuge. However, the study of the dynamical behaviour of a harvested Holling type I predator-prey system with prey refuge incorporating intraspecific competition among predator species is not treated in the existing literature, and therefore this study intended to take care of this aspect.

This paper is organized as follows: Model formulation and basic results are under the Materials and Methods section where the model is formulated and the existence of equilibrium points is studied, Boundedness of the system, stability analysis of the equilibrium points, and existence of periodic solutions are presented. The next section is about numerical solutions in which phase plots and bifurcation diagrams for examining the effects of harvesting and prey refuge to the dynamics of the prey-predator system are presented followed by discussion and concluding remarks of the work.

\section{Materials and Methods}

The mathematical model

A predator-prey system is modelled using Lotka-Voltera as follows:

$$
\begin{aligned}
& \frac{d N}{d t}=r N\left(1-\frac{N}{K}\right)-\beta_{1}(1-m) N P-H N \\
& \frac{d P}{d t}=\beta_{2}(1-m) N P-\mu P-\alpha P^{2}
\end{aligned}
$$

where $N$ and $P$ denote prey and predator population densities, respectively at any time $t$, and $r, K, \beta_{1}, \beta_{2}, \mu, \alpha$, and $H$ are all positive constants. Here $r$ represents intrinsic growth rates of prey population; $K$ is the carrying capacity; $\beta_{1}$ is the predation rate to prey species; $\mu$ is the natural mortality rate for a predator; $\beta_{2}$ is the predator biomass to the prey species; $\alpha$ is the intraspecific competition among predators; $H$ is harvesting rate in prey and $m$ is prey refuge constant with $m \in[0,1]$. The following assumptions have been taken into considerations of the model system (1): There is logistic growth of prey species in absence of predation and external factors. The rate of increase of the predator population depends on the amount of prey biomass it converts to food.

\section{Existence of equilibrium points}

To study the existence of the equilibrium points of the system (1), all possible solutions satisfying equation (2) are listed.

$$
\begin{aligned}
& r N\left(1-\frac{N}{K}\right)-\beta_{1}(1-m) N P-H N=0 \\
& \beta_{2}(1-m) N P-\mu P-\alpha P^{2}=0
\end{aligned}
$$

There is a trivial equilibrium point $E_{0}(0,0)$. The next two non-trivial equilibrium points are $E_{1}=\left(K-\frac{K H}{r}, 0\right)$ which exists if $r>H$, that is if the harvesting rate to prey population does not exceed its intrinsic growth rate, and the interior point

$$
\begin{aligned}
& E_{2}\left(\frac{\alpha K(r-H)-(\mu)\left(K m \beta_{1}-K \beta_{1}\right)}{\alpha r+\left(K m \beta_{1}-K \beta_{1}\right)\left(m \beta_{2}-\beta_{2}\right)}, \frac{K \beta_{2}(r-H)(1-m)-r(\mu)}{\alpha r+K \beta_{1} \beta_{2}(1-m)^{2}}\right) \text { which exists if } r>H \text { and } \\
& 0 \leq m<1-\frac{r(\mu)}{K \beta_{2}(r-H)} .
\end{aligned}
$$




\section{Boundedness of solution}

The state variables of model (1) represent the population densities of species. Thus, they must be non-negative at any time $t \geq 0$.

Lemma 1. All solutions of the system (1) are bounded in $\mathfrak{R}_{+}{ }^{2}=\{(N, P): N>0, P>0\}$.

Proof: Defining a function represented in Equation (3):

$S(N, P)=N+\frac{\beta_{1}}{\beta_{2}} P$

Differentiating $S$ along the trajectory; resulting into Equation (4) as:

$S^{\prime}=N^{\prime}+\frac{\beta_{1}}{\beta_{2}} P^{\prime}$

Using Equation (1) in (4) results to

$S^{\prime}(t)=(r-H) N-\frac{r N^{2}}{K}-\frac{\beta_{1}}{\beta_{2}}\left[\alpha P^{2}+(\mu) P\right]$

Taking the sum of the last relation and the Equation (3) multiplied by $v$ results into;

$$
\begin{aligned}
S^{\prime}+v S= & (r-H+v) N-\frac{r N^{2}}{K}-\frac{\beta_{1}}{\beta_{2}}\left[\alpha P^{2}+(\mu-v) P\right] \\
& \leq \frac{K}{4 r}(r-H+v)^{2}+\frac{\beta_{2}}{4 \alpha \beta_{1}}[(\mu+v)]^{2} \\
& \leq M
\end{aligned}
$$

Solving for $S$ as $t \rightarrow 0$, obtaining Equation (7) as:

$$
0 \leq S(N, P) \leq \frac{M}{v}+\left(S(0)-\frac{M}{v}\right) e^{-v t}
$$

as $t \rightarrow \infty$ gives

$$
0 \leq S(N, P) \leq \frac{M}{v}
$$

Thus, $S$ is bounded and this completes the proof.

Linear stability analysis

To study the linear stability of the equilibrium points, the Jacobian matrix of the right-hand side of Equation (1) is presented in Equation (9).

$$
J\left(E_{i}\right)=\left[\begin{array}{cc}
r\left(1-\frac{N}{K}\right)-\frac{r N}{K}-\beta(1-m) P-H & -\beta_{1}(1-m) N \\
\beta_{2}(1-m) P & \beta_{2}(1-m) N-\mu-2 \alpha P
\end{array}\right]
$$

It can be shown that the equilibrium points $E_{0}$ and $E_{1}$ are always unstable.

The equilibrium point $E_{0}$ has eigenvalues $(r-H,-\mu)$, and therefore unstable for $r>H$. The equilibrium point $E_{1}$ has unstable for $r>H$ and

$$
K \beta_{2}(1-m)\left(1-\frac{H}{r}\right)>\mu \text {. }
$$

The Jacobian matrix of the interior point $E_{2}$ takes the form;

\section{eigenvalues} $\left(H_{1}-r, K \beta_{2}(1-m)\left(1-\frac{H}{r}\right)-\mu\right)$ and

$$
J\left(E_{3}\right)=\left[\begin{array}{ll}
A_{11} & A_{12} \\
A_{21} & A_{22}
\end{array}\right]
$$


The characteristics polynomial of the matrix in Equation (10) is

$\lambda^{2}-\left(A_{11}+A_{22}\right) \lambda+\left(A_{11} A_{22}-A_{12} A_{21}\right)=0$

Where;

$A_{11}=-\left[\frac{r\left[\alpha r-\alpha H-(m-1)(\mu) \beta_{1}\right]\left[\alpha r+K(m-1)^{2} \beta_{1} \beta_{2}\right]}{\left[\alpha r+(m-1)^{2} \beta_{1} \beta_{2}\right]^{2}}\right]$,

$A_{12}=-\left[\frac{K(m-1) \beta_{1}\left[\alpha H-\alpha r+(m-1)(\mu) \beta_{1}\right]}{\alpha r+K(1-m)^{2} \beta_{1} \beta_{2}}\right]$,

$A_{21}=\frac{(m-1) \beta_{2}\left[r(\mu)+K(m-1)(r-H) \beta_{2}\right]}{\alpha r+K(1-m)^{2} \beta_{1} \beta_{2}}$,

$A_{22}=\frac{K(1-m) \alpha(r-H) \beta_{2}-\alpha r \mu}{\alpha r+K(m-1)^{2} \beta_{1} \beta_{2}}+\frac{2 \alpha r \mu-2 K(1-m) \alpha(r-H) \beta_{2}}{\left[\alpha r+K(m-1)^{2} \beta_{1} \beta_{2}\right]^{2}}+\mathrm{B}$, such that

$B=\frac{\left[\alpha^{2} r^{2}+K(m-1)^{2} \alpha r \beta_{1} \beta_{2}\right]}{\left[\alpha r+K(m-1)^{2} \beta_{1} \beta_{2}\right]^{2}}$

Since

$A_{11}<0$ and $A_{12}<0$ then, linear stability takes place if $A_{22}<0$ and $A_{21}>0$.

Existence of periodic solutions

The system of Equation (1) can be written in general form as;

$$
\begin{aligned}
& \frac{d N}{d t}=f(N, P) \\
& \frac{d P}{d t}=g(N, P)
\end{aligned}
$$

Where,

solutions if $\frac{\partial f}{\partial N}(N, P)+\frac{\partial g}{\partial P}(N, P)$ changes sign or equal to zero.

By applying this theorem to the system (1), it can be shown that
$f(N, P)=r N\left(1-\frac{N}{K}\right)-\beta_{1}(1-m) N P-H N$,

and

$g(N, P)=\beta_{2}(1-m) N P-\mu P-\alpha P^{2}$.

According to Bendixson's negative criterion, the following theorem holds:

\section{Theorem:}

The system (12) can have periodic

$\frac{\partial f}{\partial N}(N, P)+\frac{\partial g}{\partial P}(N, P) \neq 0$ and changes sign. Therefore, the system has periodic solutions.

\section{Numerical Results and Discussion}

The simulation of the model (1) is done using parameter values as given in Table 1 . 
Mapunda and Sagamiko - Mathematical analysis of harvested predator-prey system ...

Table 1: Parameter values for model (1)

\begin{tabular}{lll}
\hline Parameter & Parameter value & Source \\
\hline$r$ & 2 & Kar $(2004)$ \\
$\beta_{1}$ & 0.6 & Kar (2003) \\
$\beta_{2}$ & 0.4 & Assumed \\
$m$ & $0 \leq m<1$ & Standard range for refuge \\
$K$ & 200 & Assumed \\
$H$ & 0.24 & Das et al. 2013 \\
$\alpha$ & 0.083 & Kar and Chakraborty (2010) \\
$\mu$ & 0.0001 & Assumed \\
\hline
\end{tabular}

Bifurcation

Figure 1 shows the bifurcation behaviour of the system (1) with $m$ as a bifurcation parameter. For $m>0.78$, there is an increase in prey population and decrease in predator population leading to extinction due to resource scarcity. The decrease in predator population due to more protected prey is also described in Figure 8 .

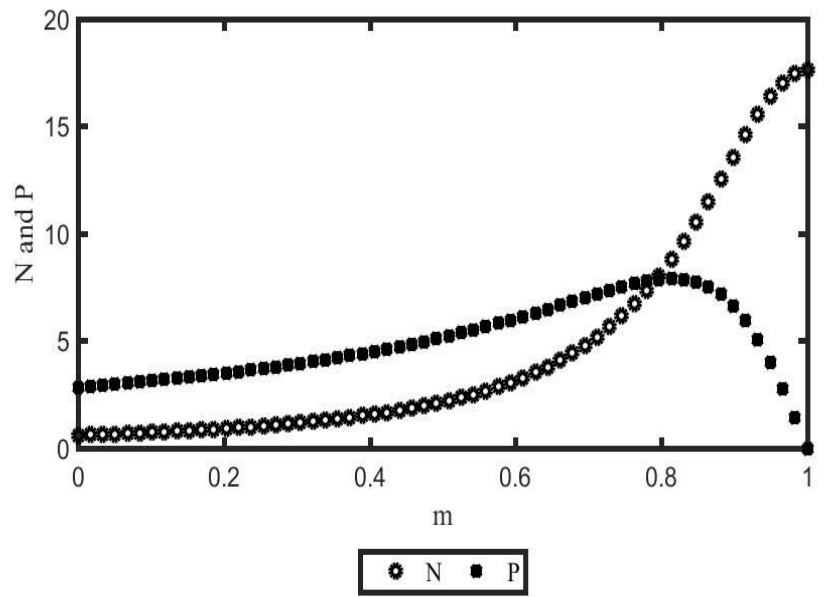

Figure 1: A bifurcation diagram for system (1) with $m$ a bifurcation parameter. The vertical axis measures both $N^{*}$ and $P^{*}$.

\section{Phase portraits}

Figure 2 shows a phase diagram that justifies the existence of periodic solutions as was done in theoretical analysis. Moreover, it is observed that the interior equilibrium point is always stable when $m=0.3$ and $H=0.3$; the behaviour which is also displayed in Figure 7. 


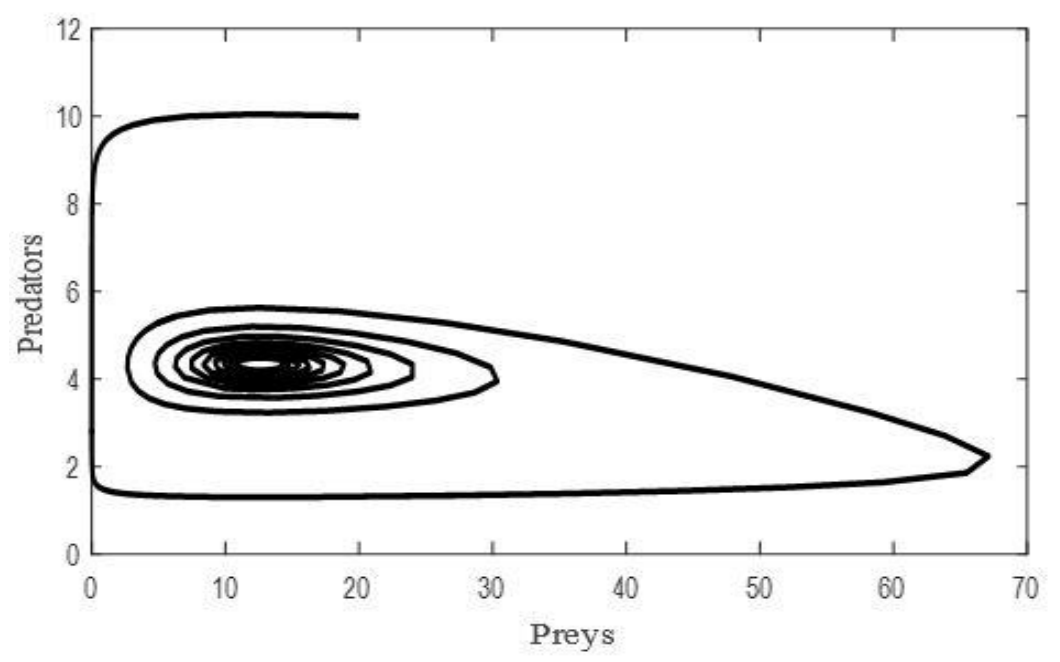

Figure 2: Phase portrait of the system (1) for $m=0.3, H=0.3$.

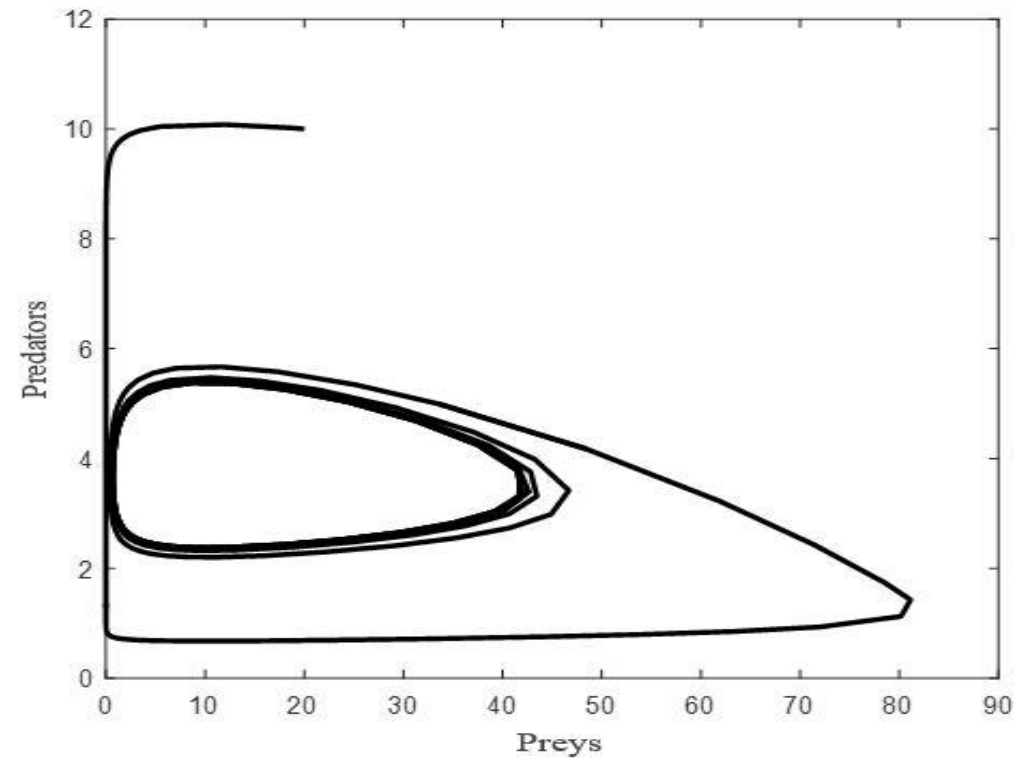

Figure 3: Phase portrait of the system (1) when $m=0.13, H=0.1$.

Figure 3 shows the effect of a decrease in refuge and harvesting. It is observed that the predator-prey system is unstable. The instability is due to excess preys resulted from small protected prey species. To bring the system into stability, the harvesting rate has to be increased as shown in Figure 4. 
Mapunda and Sagamiko - Mathematical analysis of harvested predator-prey system ...

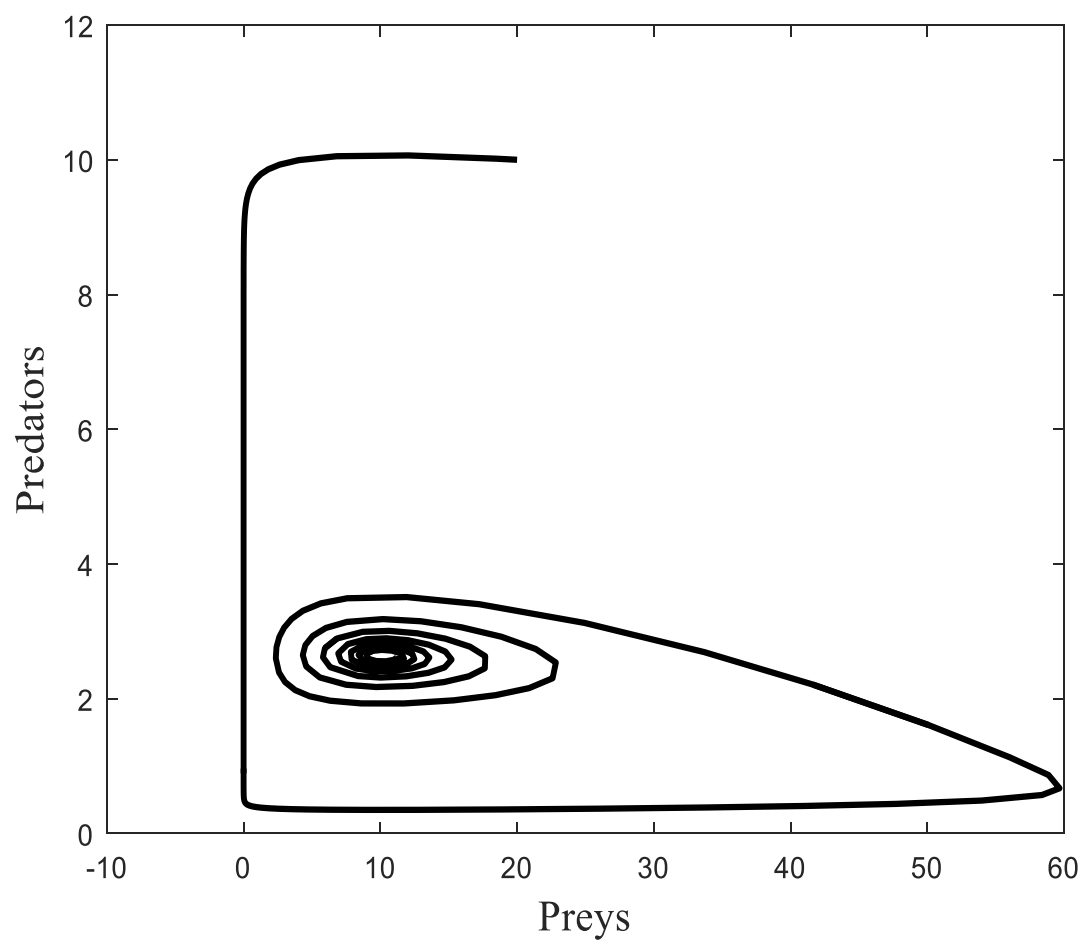

Figure 4: Phase portrait of the system (1) when $m=0.13, H=0.63$.

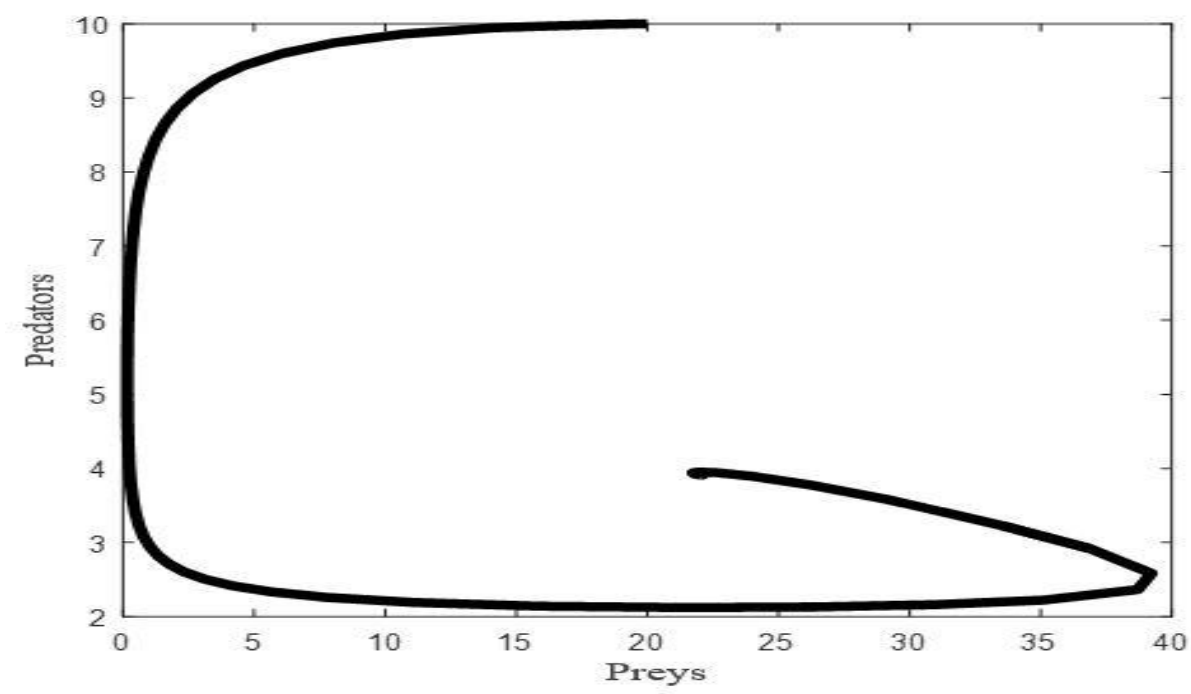

Figure 5: Phase portrait of the system (1) when $m=0.5, H=0.63$.

Figure 5 portrays the effect of increasing prey refuges for $m>0.4$ and harvesting rate above
$50 \%$ (i.e., $H=0.63$ for this case). It reveals the instability of the system which can be corrected 
by decreasing the harvesting rate to unprotected preys as shown in Figure 6.

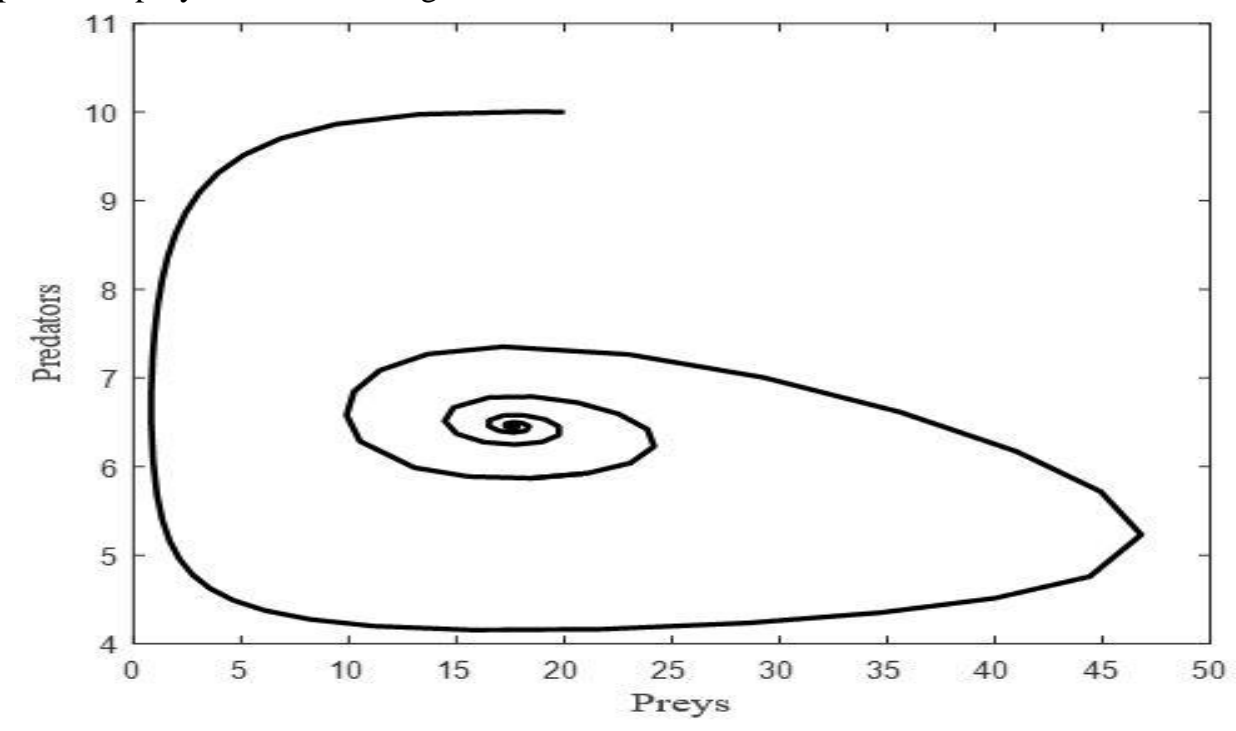

Figure 6: Phase portrait of the system (1) when $m=0.5, H=0.01$.

Species interactions

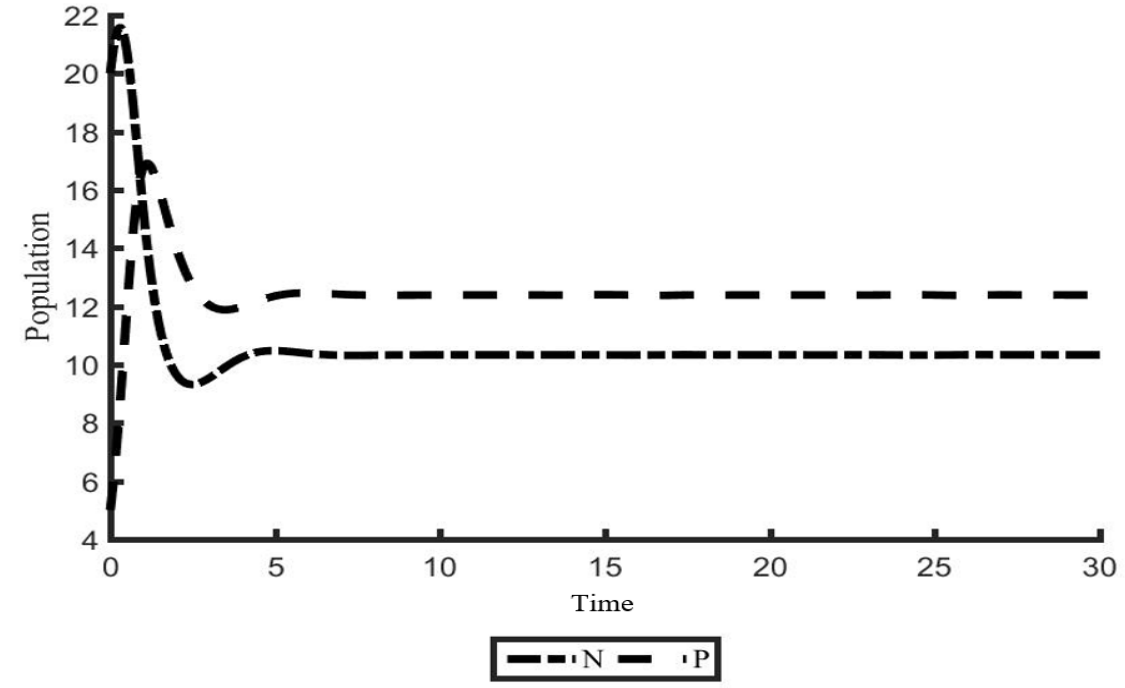

Figure 7: Predator-prey interaction for protected prey $m<0.3$. 
Mapunda and Sagamiko - Mathematical analysis of harvested predator-prey system ...

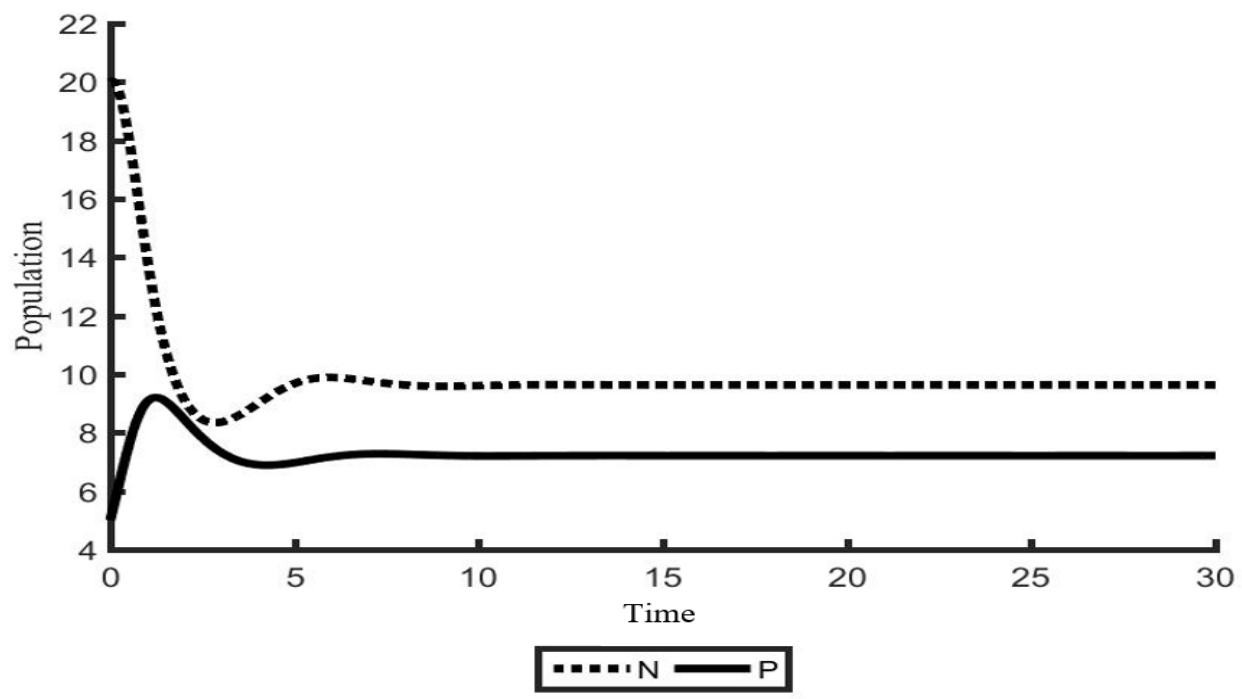

Figure 8: Predator-prey interaction for increased protected prey $m>0.5$.

Prey harvesting

Figure 9 demonstrates the extinction of both species when the rate of harvesting exceeds prey intrinsic growth rate. This results remind

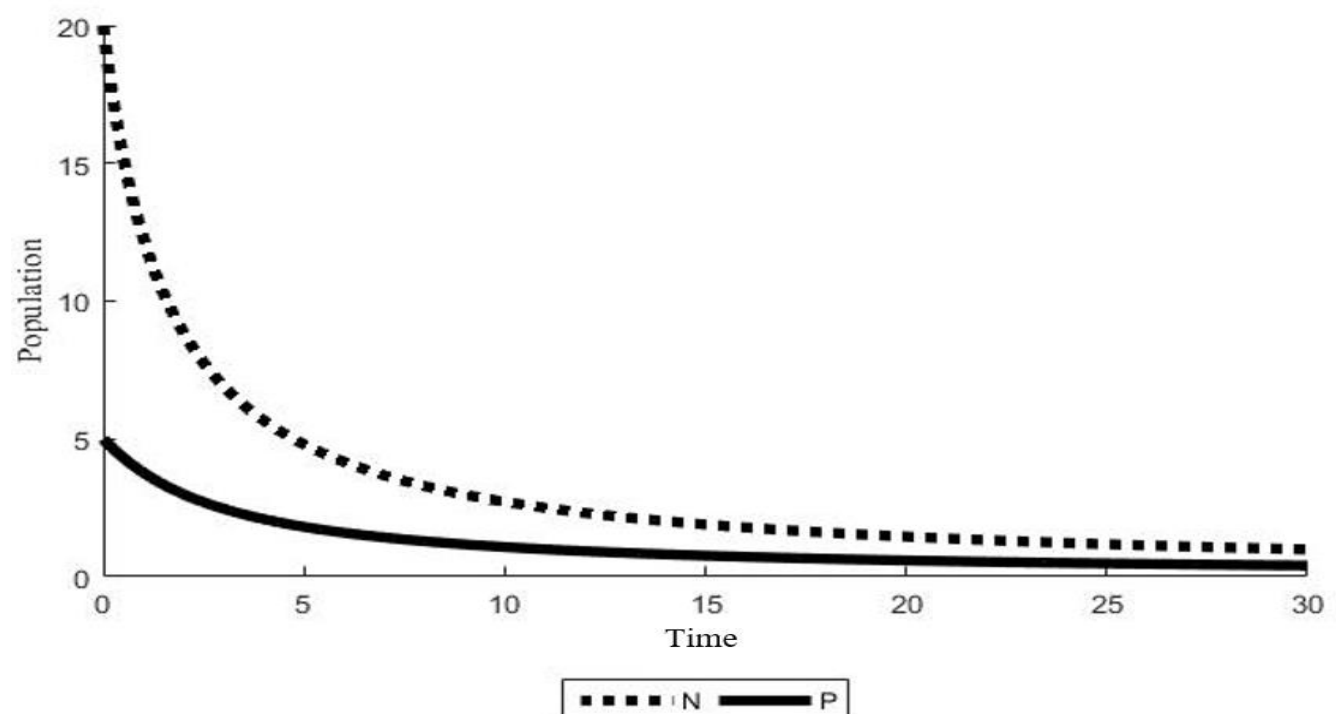

Figure 9: Effect of harvesting on prey density when $H \geq r$. of the need for abiding with the theoretical analysis that the harvesting rate should not exceed prey intrinsic growth rate for the predator-prey system to be stable. 
Tanz. J. Sci. Vol. 47(2), 2021

\section{Conclusion}

In this work, a dynamic behaviour of a harvested predator-prey system with prey refuge and intraspecific competition was studied. The model incorporated harvesting to prey and intraspecific competitions to predator individuals. It was also assumed that Holling type I is the predator's functional response to preys. Numerical studies have been conducted to verify the theoretical results. The dynamical behaviour indicated by simulations is in agreement with the theoretical results.

\section{Acknowledgements}

Authors acknowledge their institutions for the provision of research resources.

Declaration: The authors have no conflict of interest.

\section{References}

Berryman AA 1992 The origins and evolution of predator-prey theory. Ecol.73: 15301535.

Das U, Kar TK and Pahari UK 2013 Global dynamics of an exploited prey-predator model with constant prey refuge. Int Scholarly Res. Notices Biomathematics 2013: $12 \mathrm{p}$.

Dawes JHP and Souza MO 2013 A derivation of Holling's type I, II and III functional responses in predator-prey systems. $J$. Theor. Biol. 327(1): 11-22.

Kar TP 2005 Stability and analysis of preypredator model incorporating a prey refuge.
Commun. Nonlinear Sci. Numer. Simul. 10: 681-691.

Kar TP 2006 Modelling and analysis of a harvested prey-predator system incorporating a prey refuge. J. Comput. Appl. Math. 185: 19-33.

Kar TP and Chakraborty 2010 Effort dynamics in a prey-predator model with harvesting. Int. J. Inf. Syst. Sci. 6(3): 318-332.

Majeed AA 2018 The dynamics of preypredator model with prey refuge and stage structures in both populations. Sci. Int. (Lahore). 30(3): 461-470.

Mapunda A, Mureithi E, Shaban N and Sagamiko T 2018 Effects of overharvesting and drought on a predator-prey system with optimal control. Open J. Ecol. 459-482.

Sagamiko TD, Shaban N, Nahonyo CL and Makinde OD 2015 Optimal control of a threatened wildebeest-lion prey-predator system incorporating a constant prey refuge in the Serengeti ecosystem. Appl. Comput. Math. 4(4): 296-312.

Schwinning S and Kelly CK 2013 Plant competition, temporal niches and implications for productivity and adaptability to climate change in waterlimited environments. Funct. Ecol. 27: 886897.

Stefanou I and Alevizos S 2016 Fundamentals of bifurcation theory and stability analysis. Instabilities Modeling in Geomechanics, pp. 31-71, John Wiley \& Sons, Inc. 